\title{
ANALYSIS OF CARDANOL AS A SUBSTITUTE FOR DIESEL
}

\author{
Chandrasekaran.S \\ Assistant professor Vels University, Chennai, India.
}

\begin{abstract}
The demand for alternate fuels used in the operation of I.C engine is met with CNSL (Cashew nut shell liquid) a by-product of cashew industry which is considered as an economically suitable for producing biodiesel. Cashew nut shell liquid is obtained from the hard cashew nut shell, cardinal a natural phenol obtained from the pyrolysis of CNSL at reduced pressure. The viscosity of the cardinal is very high compared to that of diesel. The parametric characteristic of I.C engines with various injection timing and pressure using fuel as Cashew nut shell oil is found. Cashew nut shell liquid is being used in our Defence force heavy truck vehicle-SHAKTHIMAN.
\end{abstract}

\section{INTRODUCTION}

\subsection{Need for Alternate fuel}

The depleting reserves of fossil fuels, increasing demand for diesel an alternate source in their availability has emerged as initiatives to search for alternate sources of energy, so it can be supplement or replace fossil fuels. At present years, rather than using fossil fuels, researchers have focused their upon using the products of plant that is seed oil and fats extracted from plants, in that way the most commonly used alternated fuels for now is bio-diesel. Which is made up of fatty acid methyl esters(FAMEs) from oil seeds and fats. FAMEs bio-diesel is economically safe, non-toxic and biodegradable .Bio-diesel producing countries use edible fatty oils derived from rapeseed ,soybean, palm, sunflower, coconut, linseed etc., as a raw material but such types of edible oils are not possible to be used in India, due to the increasing supply and demand of these oils in India. Thus the increase in demand of such oils has put a stop for these oils to be used in making of Bio diesel. In a country like India, the use of only non edible oil is promoted for making Bio diesel in large quantity and planted on large scale on non cropped and waste lands.

\subsection{Cashew Nut Shell Liquid}

There are a large number of trees, shrubs and herbs which are present in India and that can be used as a Bio diesel fuel. Nearly $30-35 \%$ CNSL is available in the shell where shell percentage $67 \%$ of nut. The cashew processing industries produce CNSL as a byproduct. Cashew nut shell undergoes various ethnical processing to obtain CNSL. At first, the heat obtained CNSL, the heating process leads to decarboxylation of anacardic acid to form Cardanol, this comprises of $10 \%$ cardol and $30 \%$ polymeric material and the remaining percentage is made up of substances. After heating process, distillation is done at a reduced pressure inorder to remove the polymeric material. Now the distilled CNSL consists of 78\% Cardanol , $8 \%$ cardol, $2 \%$ polymeric material 
and remaining other substances. At this present commercial extraction process is carried out by boiling or heating CNSL upto 108 degree centigrade to obtain cardanol rich oil.

\subsection{Cardanol}

Cardanol is obtained from cashew Nut shell Liquid (CNSL) - a Monohydroxyl phenol having hydrocarbon chain in the Meta position.

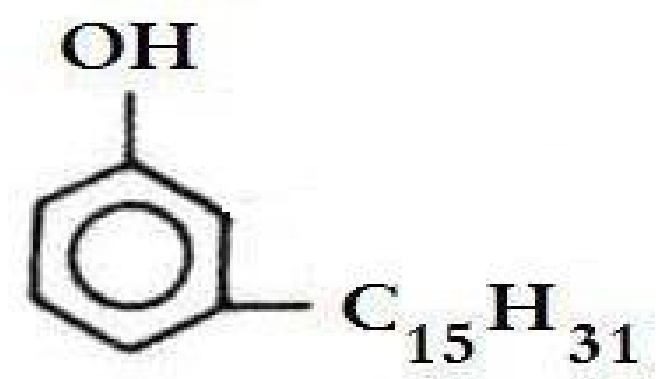

Figure 1: Structure of Cardanol

Cardanol is processed and extracted from CNSL with respective its fractional distillation at $200^{\circ} \mathrm{C}$ to $240^{\circ} \mathrm{C}$ with a pressure less than $5 \mathrm{~mm}$ of mercury. Cardanol is renewable, cost effective and easily produced in many regions in India and Asian countries with the limited usage of diesel. These products extracted from Cardanol have many advantages over other substituted phenols. Therefore it is widely used in the manufacture of surface coatings, Insulating varnishes, oil and alcohol soluble resins, laminating resins, Rubber compounding, Azo dyes, etc. As the viscosity of Cardanol is very high (compared to diesel) in the present investigation.

\section{RELATED WORK}

A number of different oils have been investigated in recent years and their performances and emission characteristics have been compared with Diesel. Kasiraman et al [5] report that a blend of $70 \%$ CNSL and 30\% camphor Oil give results similar to Diesel. Velmurugan et al [11] tested of commercial diesel fuel and biofuel from CNSL with ethanol as an additive (BDEB). Experiemental Investigation of CNSL as alternative was under taken by V.Palvannan et al and the first trial run with a maximum of $20 \%$ CNSL- diesel blend was successfully conducted on single cylinder CI engine. engine power output and smoke emissions are found to be satisfactory,then it was decided to to proceed further testing. Mallikappa et al [7] investigated the performance and emission characteristics with $0.5 \%, 10 \%, 15 \%, 20 \%$ and $25 \%$ blends of Cardanol and Diesel. They reported that up to $20 \%$ blends of cardinal bio fuels may be used in CI engines without any modifications. 
Table 1:Fuel Properties

\begin{tabular}{|c|c|c|c|}
\hline Sl. No. & \multicolumn{1}{|c|}{ Tests } & Cardanol Oil ( CNSL ) & Diesel \\
\hline 1 & Density @ $15^{\circ} \mathrm{C} \mathrm{(} \mathrm{in} \mathrm{gm/cc)}$ & 0.9331 & 0.84 \\
\hline 2 & Kinematic Viscosity @ $40^{\circ}$ & $27.80 \mathrm{cst}$ & 4.59 \\
\hline 3 & Flash Point by PMCC method & $168^{\circ} \mathrm{C}$ & $50^{\circ} \mathrm{C}$ \\
\hline 4 & Fire Point by PMCC method & $182^{\circ} \mathrm{C}$ & $68^{\circ} \mathrm{C}$ \\
\hline 5 & Gross Calorific value in Kcal/Kg & 9651 & 10152.89 \\
\hline 6 & Conradson Carbon Residue & $0.55 \%$ & $\cdots$ \\
\hline
\end{tabular}

\subsection{Testing setup of an engine}

The diesel engine is a high rpm with four strokes, vertical and air cooled type. Loading by means of an electrical dynamometer. To find out the how much quantity of fuel is consumed per unit time is measured with the help of graduated burette which is located outer end of the fuel tank .An orifice meter with $U$ tube manometer provided along with an air tank on suction line for measuring air consumption. An AVL 4.5 smoke meter is provided for measuring a FSN of exhaust gases. The rig is installed with AVL software for obtaining various curves and results during operation. A five gases and analyzer are used to obtain the exhaust gas combustion.

\subsection{Engine specification}

Table 2.1 Engine Specification

\begin{tabular}{|c|l|l|}
\hline Sl. No. & \multicolumn{1}{|c|}{ Description } & \multicolumn{1}{c|}{ Specification } \\
\hline 1 & Engine make & Kirloskar oil engine \\
\hline 2 & Engine model & TAF 1 \\
\hline 3 & Engine type & $\begin{array}{l}\text { Four stroke, single cylinder, } \\
\text { vertical, air cooled diesel engine. }\end{array}$ \\
\hline 4 & Rated power & $4.4 \mathrm{~kW}$ \\
\hline 5 & Rated speed & $1500 \mathrm{rpm}$ \\
\hline 6 & Bore diameter ( D ) & $87.5 \mathrm{~mm}$ \\
\hline 7 & Stroke ( L ) & $110 \mathrm{~mm}$ \\
\hline 8 & Compression ratio & $17.5: 1$ \\
\hline 9 & Orifice diameter & $13.4 \mathrm{~mm}$ \\
\hline 10 & Coefficient of discharge & 0.62 \\
\hline
\end{tabular}




\section{RESUlts AND DisCUSSION}

The performance of emission \& combustion test have been conducted on $4.4 \mathrm{~kW}$ constant speed diesel engine.The parametric study has been conducted using Cardanol as fuel.The parametric study have been conducted at various injector pressure $180,200,220 \& 240$ bar \& timing $21^{\circ}, 23^{\circ}, 25^{\circ} \& 27^{\circ}$ bTDC.Based on the experimental observations the following conclusions were drawn. At peak load among the combinations of injection parametric study $25^{\circ}$ bTDC \& 240 bar operations gives the best efficiency on par with Diesel. The summary results are presented.

\subsection{Performance Characteristics}

Table 3: Neat Cardanol at Injection timing $25^{\circ}$ bTDc

Diesel at Injection timing $23^{\circ} \mathrm{bTDC}$ and injection pressure $200 \mathrm{bar}$

\begin{tabular}{|c|c|c|c|c|c|c|c|c|c|c|c|c|c|c|c|c|c|c|}
\hline \multirow{5}{*}{ Fuel used } & \multirow{4}{*}{$\begin{array}{c}\text { Injection } \\
\text { timing }\end{array}$} & \multirow{4}{*}{ Load } & \multicolumn{4}{|c|}{ Brake thermal efficiency } & \multicolumn{4}{|c|}{ Brake power } & \multicolumn{4}{|c|}{ Specific fuel consumption } & \multicolumn{4}{|c|}{ Carbon monoxide } \\
\hline & & & \multicolumn{4}{|c|}{$\%$} & \multicolumn{4}{|c|}{ KW } & \multicolumn{4}{|c|}{$\mathrm{Kg} / \mathrm{KWhr}$} & \multicolumn{4}{|c|}{$\%$} \\
\hline & & & \multicolumn{4}{|c|}{$\eta B T$} & \multicolumn{4}{|c|}{$\mathrm{BP}$} & \multicolumn{4}{|c|}{ SFC } & \multicolumn{4}{|c|}{$\mathrm{CO}$} \\
\hline & & & \multicolumn{4}{|c|}{ Injection pressure } & \multicolumn{4}{|c|}{ Injection pressure } & \multicolumn{4}{|c|}{ Injection pressure } & \multicolumn{4}{|c|}{ Injection pressure } \\
\hline & deg & $\%$ & 180 & 200 & 220 & 240 & 180 & 200 & 220 & 240 & 180 & 200 & 220 & 240 & 180 & 200 & 220 & 240 \\
\hline \multirow{5}{*}{$\begin{array}{c}\text { NEAT } \\
\text { CARDANOL }\end{array}$} & $25^{\circ}$ & 0 & 0 & 0 & 0 & 0 & 0 & 0 & 0 & 0 & 0 & 0 & 0 & 0 & 0.17 & 0.13 & 0.24 & 0.13 \\
\hline & $25^{\circ}$ & 25 & 13.691 & 14.603 & 13.064 & 14.254 & 1.170 & 1.170 & 1.165 & 1.165 & 0.651 & 0.610 & 0.682 & 0.625 & 0.13 & 0.10 & 0.11 & 0.10 \\
\hline & $25^{\circ}$ & 50 & 20.052 & 21.543 & 20.052 & 21.825 & 2.280 & 2.290 & 2.280 & 2.270 & 0.444 & 0.414 & 0.444 & 0.408 & 0.10 & 0.06 & 0.08 & 0.08 \\
\hline & $25^{\circ}$ & 75 & 24.837 & 25.175 & 24.115 & 26.461 & 3.360 & 3.360 & 3.360 & 3.330 & 0.359 & 0.354 & 0.369 & 0.337 & 0.09 & 0.08 & 0.11 & 0.08 \\
\hline & $25^{\circ}$ & 100 & 23.344 & 23.706 & 23.989 & 24.268 & 4.340 & 4.360 & 4.340 & 4.320 & 0.382 & 0.376 & 0.371 & 0.367 & 0.38 & 0.24 & 0.45 & 0.42 \\
\hline
\end{tabular}


International Journal of Managing Value and Supply Chains (IJMVSC) Vol. 7, No. 2, June 2016

Table 4.Diesel and Cardanol

\begin{tabular}{|c|c|c|c|c|c|c|c|c|c|c|c|c|c|c|c|c|c|c|c|c|c|}
\hline \multirow{4}{*}{$\begin{array}{l}\text { Injection } \\
\text { timing }\end{array}$} & \multirow{4}{*}{ Load } & \multicolumn{4}{|c|}{ Hydro carbon } & \multicolumn{4}{|c|}{ Carbon di oxide } & \multicolumn{4}{|c|}{ Oxygen } & \multicolumn{4}{|c|}{ Nitrogen oxides } & \multicolumn{4}{|c|}{ Filter smoke number } \\
\hline & & \multicolumn{4}{|c|}{ PPM } & \multicolumn{4}{|c|}{$\%$ in Vol } & \multicolumn{4}{|c|}{$\%$ in Vol } & \multicolumn{4}{|c|}{ PPM } & & & & \\
\hline & & \multicolumn{4}{|c|}{ HC } & \multicolumn{4}{|c|}{$\mathrm{CO} 2$} & \multicolumn{4}{|c|}{02} & \multicolumn{4}{|c|}{ NOX } & \multicolumn{4}{|c|}{ FSN } \\
\hline & & \multicolumn{4}{|c|}{ Injection pressure } & \multicolumn{4}{|c|}{ Injection pressure } & \multicolumn{4}{|c|}{ Injection pressure } & \multicolumn{4}{|c|}{ Injection pressure } & \multicolumn{4}{|c|}{ Injection pressure } \\
\hline $\operatorname{deg}$ & $\%$ & 180 & 200 & 220 & 240 & 180 & 200 & 220 & 240 & 180 & 200 & 220 & 240 & 180 & 200 & 220 & 240 & 180 & 200 & 220 & 240 \\
\hline $25^{\circ}$ & 0 & 26 & 16 & 36 & 26 & 2.0 & 1.9 & 2.3 & 1.9 & 17.93 & 18.13 & 17.42 & 18.05 & 68 & 123 & 45 & 121 & 0.29 & 0.26 & 0.13 & 0.03 \\
\hline $25^{\circ}$ & 25 & 25 & 14 & 21 & 18 & 2.9 & 2.8 & 3.2 & 2.9 & 16.74 & 16.85 & 16.13 & 16.8 & 244 & 282 & 222 & 293 & 0.49 & 0.55 & 0.46 & 0.44 \\
\hline $25^{\circ}$ & 50 & 26 & 16 & 18 & 17 & 3.7 & 3.7 & 4.3 & 4.2 & 15.3 & 15.65 & 14.83 & 14.96 & 541 & 553 & 508 & 583 & 1.14 & 1.40 & 1.69 & 0.96 \\
\hline $25^{\circ}$ & 75 & 25 & 20 & 18 & 18 & 5.1 & 5.7 & 5.5 & 5.4 & 13.9 & 13.09 & $13.27 \mid$ & 13.26 & 820 & 889 & 610 & 759 & 1.44 & 2.37 & 1.84 & 1.56 \\
\hline $25^{\circ}$ & 100 & 39 & 28 & 33 & 27 & 6.0 & 7.0 & 7.5 & 8.2 & 12.25 & 11.23 & 10.35 & 9.65 & 629 & 710 & 599 & 664 & 4.11 & 3.49 & 4.53 & 3.59 \\
\hline
\end{tabular}

\begin{tabular}{|c|c|c|c|c|c|c|c|c|c|c|c|}
\hline \multirow{5}{*}{ Fuel used } & \multirow{4}{*}{$\begin{array}{c}\text { Injection } \\
\text { timing }\end{array}$} & \multirow{4}{*}{ Load } & $\begin{array}{c}\text { Brake } \\
\text { thermal } \\
\text { efficiency }\end{array}$ & $\begin{array}{c}\text { Brake } \\
\text { power }\end{array}$ & $\begin{array}{l}\text { Specific fuel } \\
\text { consumption }\end{array}$ & $\begin{array}{c}\text { Carbon } \\
\text { monoxide }\end{array}$ & $\begin{array}{l}\text { Hydro } \\
\text { carbon }\end{array}$ & $\begin{array}{l}\text { Carbon di } \\
\text { oxide }\end{array}$ & Oxygen & $\begin{array}{c}\text { Nitrogen } \\
\text { oxides }\end{array}$ & $\begin{array}{c}\text { Filter } \\
\text { smoke } \\
\text { number }\end{array}$ \\
\hline & & & $\%$ & KW & $\mathrm{Kg} / \mathrm{KWhr}$ & $\%$ & PPM & $\%$ in Vol & $\%$ in Vol & PPM & \\
\hline & & & $\eta B T$ & $\mathrm{BP}$ & SFC & co & $\mathrm{HC}$ & $\mathrm{CO} 2$ & 02 & NOX & FSN \\
\hline & & & \multicolumn{9}{|c|}{ Injection pressure } \\
\hline & $\operatorname{deg}$ & $\%$ & \multicolumn{9}{|c|}{200} \\
\hline \multirow{7}{*}{ DIESEL } & $23^{\circ}$ & 0 & 0 & 0 & 0 & 0.14 & 31 & 2.0 & 17.91 & 148 & 0.22 \\
\hline & $23^{\circ}$ & 25 & 14.723 & 1.165 & 0.605 & 0.08 & 22 & 2.9 & 16.6 & 378 & 0.56 \\
\hline & $23^{\circ}$ & 50 & 22.108 & 2.270 & 0.403 & 0.07 & 25 & 4.0 & 15.12 & 689 & 1.31 \\
\hline & $23^{\circ}$ & 75 & 25.098 & 3.345 & 0.355 & 0.08 & 28 & 5.7 & 12.84 & 1255 & 1.92 \\
\hline & $23^{\circ}$ & 100 & 24.600 & 4.320 & 0.362 & 0.23 & 36 & 7.2 & 10.92 & 1004 & 2.96 \\
\hline & & & \multicolumn{9}{|c|}{ Injection pressure } \\
\hline & $\operatorname{deg}$ & $\%$ & \multicolumn{9}{|c|}{240} \\
\hline \multirow{5}{*}{$\begin{array}{c}\text { NEAT } \\
\text { CARDANOL }\end{array}$} & $25^{\circ}$ & 0 & 0 & 0 & 0 & 0.13 & 26 & 1.9 & 18.05 & 121 & 0.03 \\
\hline & $25^{\circ}$ & 25 & 14.254 & 1.165 & 0.625 & 0.10 & 18 & 2.9 & 16.8 & 293 & 0.44 \\
\hline & $25^{\circ}$ & 50 & 21.825 & 2.270 & 0.408 & 0.08 & 17 & 4.2 & 14.96 & 583 & 0.96 \\
\hline & $25^{\circ}$ & 75 & 26.461 & 3.330 & 0.337 & 0.08 & 18 & 5.4 & 13.26 & 759 & 1.56 \\
\hline & $25^{\circ}$ & 100 & 24.268 & 4.320 & 0.367 & 0.42 & 27 & 8.2 & 9.65 & 664 & 3.59 \\
\hline
\end{tabular}




\section{CoMparison BeTWeEn Diesel VS NeAT CARdANOL}

Graph-1

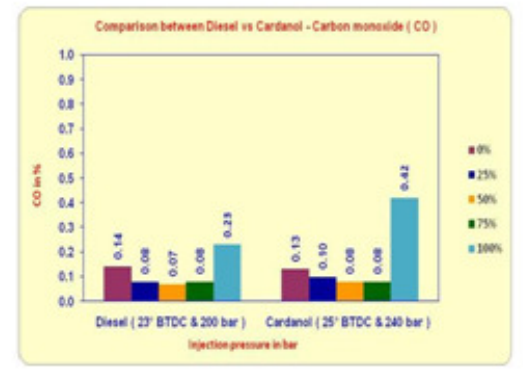

Graph-3

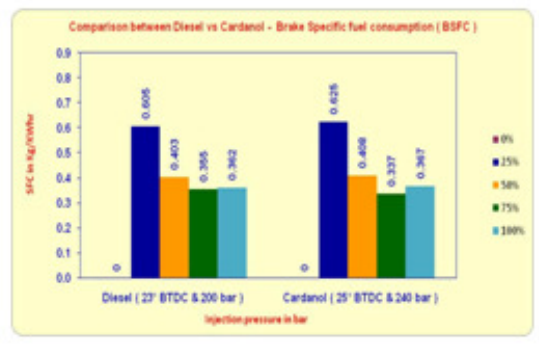

Graph-2

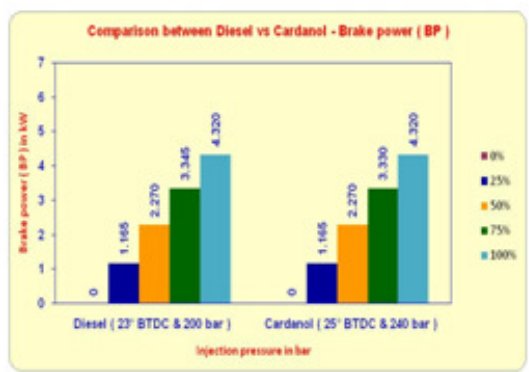

Graph-4

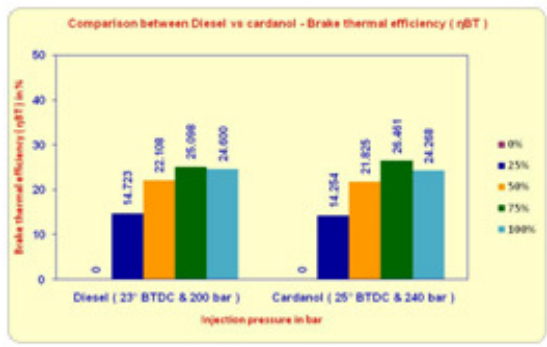

\section{Conclusion}

The CNSL oil is most useful non edible for the yield of bio diesel. Cardanol oil is used to used to run the multifuel engine which is extracted from the raw CSNL oil . Based on the experimental investigations carried out on the IC engine ,Cardanol oil can be used as alternative for diesel. From the experimental results it is recommended to use Cardanol $25^{\circ}$ bTDC and 240 bar for improved performance.

\section{REFERENCES}

[1] Piyali Das, Sreelatha.T \& Ganesh.A (2004). Bio oil from pyrolysis of cashew nut shell Characterization and related properties. Biomass and Bioenergy 27, 265-275.

[2] Gumus.M, Sayin.C and Canakci.M (2012 ).the impact of fuel injection pressure on the exhaust emission of the direct injection diesel engine fueled with biodiesel - Diesel fuel blend. Fuel 95, 486494.

[3] Harrington, (1986). Chemical and physical properties of vegetable oil esters and their effect on diesel fuel performance. Biomass, 9:1- 17.

[4] Jindal.S, Nandwana.D, Rathore.N \& Vashistha.V (2010). Experimental investigation of the effect of compression ratio and injection pressure in a direct injection diesel engine running on Jatropha methyl ester. Applied thermal engineering 30,442-448.

[5] Kasiraman.G, Nagalingam.B and Balakrishnan.N (2012). Performance, emission and combustion improvements in a direct injection diesel engine using cashew nut shell oil as fuel with Camphor oil blending. Energy 47,116-124.

[6] Korbitz.W (1999). Biodiesel production in Europe and North America, an encouraging prospect. Renewable energy, 16:1078-83. 
International Journal of Managing Value and Supply Chains (IJMVSC) Vol. 7, No. 2, June 2016

[7] Mallikappa.D, Reddy.R.P and Murthy.C.S (2012). Performance and emission characteristics of double cylinder CI engine with Cardanol biofuel blends. Renewable energy 38,150-154.

[8] Martini.N, Shell.J and Editors (1998). Plant oils as fuels - Present state of Science and future development. Berlin: Springer, 276.

[9] Puhan.S, Jagan.R, Balasubbramanian.K and Nagarajan.G (2009). Effect of injection pressure on performance, emission and combustion characteristics of high Linolenic linseed oil methyl ester in a DI diesel engine. Renewable energy 34, 1227-1233.

[10] Purushothaman.K and Nagarajan.G (2009). Effect of injection pressure on heat release rate and emission in CI engine using Orange skin powder diesel solution. Energy conversion and management 50, 962-969.

[11] Velmurugan.A and Loganathan.M (2011). Performance and emission characteristics of a DI diesel engine fuelled with cashew nut shell liquid (CNSL) - Diesel blends. World Academy of science, Engineering and technology 58.

\section{Author}

S. Chandrasekaran, M.Tech( $\left.2^{\text {nd }} \mathrm{yr}\right)$, Bharath University, Chennai.

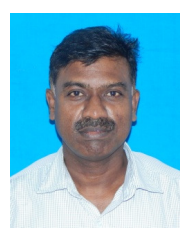

